\title{
Stress-driven MEMS Assembly + Electrostatic Forces = 1mm Diameter Robot
}

\author{
Mustafa Emre Karagozler, Seth Copen Goldstein, J. Robert Reid
}

\begin{abstract}
As the size of the modules in a self-reconfiguring modular robotic system shrinks and the number of modules increases, the flexibility of the system as a whole increases. In this paper, we describe the manufacturing methods and mechanisms for a 1 millimeter diameter module which can be manufactured en masse. The module is the first step towards realizing the basic unit of claytronics, a modular robotic system designed to scale to millions of units.
\end{abstract}

\section{INTRODUCTION}

As the size of the modules in a self-reconfiguring modular robotic system shrinks and the number of modules increases, the flexibility of the system as a whole increases. In this paper, we describe the manufacturing methods and mechanisms for a 1 millimeter diameter module which can be mass produced. The module, which we call a catom, is the basic unit for claytronics, a modular robotic system designed to scale to millions of units [9].

Successfully realizing claytronics rests firmly on the ability to mass-produce sub-millimeter scale catoms. Each catom must have a small robust physical structure integrated with the ability to transfer energy to neighboring units, store energy, move, communicate with its neighbors and selectively adhere to neighboring units; all under the local control. To invest each unit with all of this functionality requires a novel approach towards the design and manufacture of the catom. Our design philosophy seeks to simplify the catom design by following the ensemble principle, which states that: A unit should include only enough functionality to contribute to the desired functionality of the ensemble. Adherence to the ensemble principle forces us to use local computation and nearest-neighbor communication to simplify the physical mechanisms in each catom as much as possible. In this instance, we use one physical mechanism (conductive plates on, or near, the surface of the unit) for locomotion, adhesion, communication, and power transfer. The local control of the catom has the ability to move charge between the plates. This charge generates electrostatic forces between the robots to accomplish all the aforementioned functions.

The simple mechanism makes mass production of the robot possible using a combination of photolithography,

This work was supported in part by NSF Grants CNS-0428738, ITR0326054, AFRL, and Intel Corporation.

Karagozler in Electrical and Computer Engineering at Carnegie Mellon University, 5000 Forbes Ave, Pittsburgh, PA 15213 mkaragozeandrew.cmu.edu. Goldstein in Computer Science Department at Carnegie Mellon University seth@cs.cmu.edu. Reid at Air Force Research Laboratory, Hanscom AFB, MA James.Reid@hanscom.af.mil.

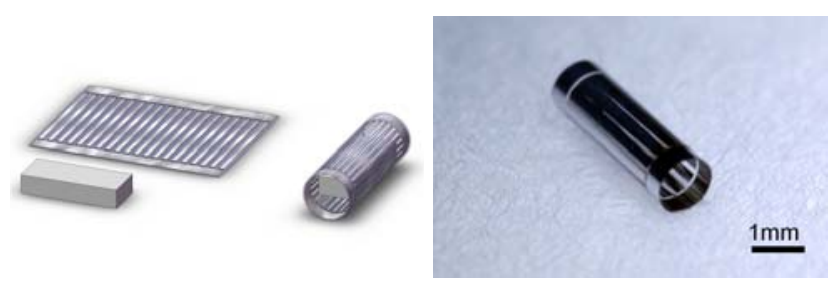

Fig. 1. The Catom: Tube + Chip.

Fig. 2. Fabricated Catom.

pick-and-place technology, and stress-driven assembly. Photolithography is used to create a die with the circuits for local control and the metal plates. The resulting die are designed so that when released from the wafer they curl up into the 3D shape of the unit. Eventually this process will result in a spherical catom with all of the required functionality. Achieving this goal will obviously require more than one design iteration. In this paper, we detail the implementation of an early generation catom at the millimeter scale (See Fig. 1 and Fig. 2). We detail the process for forming three dimensional shapes from two dimensional patterns, details of the electrostatic actuation, and our on-going work to implement energy transfer and internal actuation. The paper closes with a discussion of our current status and the next steps in the process.

\section{A. Our Research Path}

The design and implementation of a sub-millimeter autonomous robot, even one which only needs to function in an ensemble, is challenging and therefore will be done over several design generations. The first generation, detailed here, aims to develop the fabrication process and implement the core functionality of the catoms: manufacturing, energy transfer, energy storage, and actuation. Once this is achieved, functionality can be added to the catom through relatively minor process improvements and adding additional circuitry.

Our approach, motivated by the need to mass produce the robots, is to integrate as many of the individual components as possible. Our first design decision was to make each robot a sphere. This simplifies manufacturing, while providing significant flexibility in the lattice arrangement of the individual modules. As described in [14], [15], a sphere can be created through stress-driven assembly of a photolithographically defined die. Locomotion is achieved by having the robots orbit each other using electrostatic forces as described in Section III. These electrodes can be readily fabricated in two dimensions before the device folds up. Having a solid approach towards realizing the structure, the next step is to integrate active circuitry into the catoms. In order to 
simplify manufacturing and assembly challenges, we decided to focus on integration and temporarily work on cylindrical tubes rather than spheres. Using tubes relaxes the spatial constraints on interior components and allows us to focus on improving the folding process to enable regions with changing radii (see Section II-A), design of the actuators, and integration of power transfer and active circuitry. Note that all of the techniques developed are directly applicable to the final spherical shape so that simply decreasing the driver circuit size and changing the initial two dimensional pattern will enable us to move from the tube back to a spherical shape. The unit we present here represents all of the core capability required in a catom so that moving forward is a matter of implementing new circuits and adding functionality to the die.

Following the ensemble principle, we use conductive plates near the surface of the catom to provide actuation [11]. A first generation circuit capable of rectifying an incoming $\mathrm{AC}$ signal and actuating the catom has been designed. Testing of the circuit has shown promise, but several critical components require a redesign. These circuits are implemented using a commercial CMOS foundry.

\section{B. Related Work}

Previous work in microscale ( $\leq 1 \mathrm{~mm}$ on a side) robots has generally focused on externally controlled and actuated units which only move in the plane. For example, Floyd et.al. [6] and Frutiger et.al. [7] use externally generated magnetic fields to actuate micrometer sized magnetic pieces on a 2D plane. Fujita et.al. [8] using principles similar to those described in this paper demonstrated electrostatic actuation to rotate conducting cylinders.

While we are the first to propose a monolithically manufactured non-planar robot that can move out of the plane, Donald et al. [5] demonstrated a planar robot which was fabricated using photolithography. They use PolyMUMPS to fabricate a sub-millimeter scale planar root with a stress engineered steering arm. The robot utilizes an electrostatic stick-slip actuator, which operates on a powered floor. Hollar et.al. [10] demonstrated a solar powered microrobot which is fabricated by assembling a MEMS-micromotor, a high voltage chip and a CMOS chip.

\section{Manufacturing The Tube Microrobot}

\section{A. Fabrication of the Tube}

The process for fabricating the catoms is critical to the goal of creating ensembles of thousands to millions of them. Densely packed functionality must be provided through highly repeatable low-cost mass production. Therefore, the process we are developing is based around commercial CMOS processes. These processes are ideal for producing large numbers of repeatable, compact, highly integrated circuits.

In the long run, our fabrication process will have three distinct steps: CMOS foundry fabrication, pick and place bonding, and three dimensional micromachining. The first two steps in this process are both widely available from

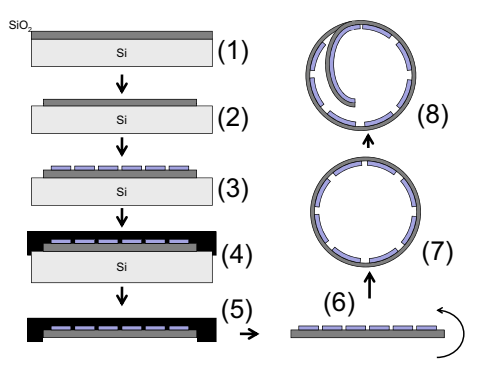

Fig. 3. The process steps for catom fabrication.

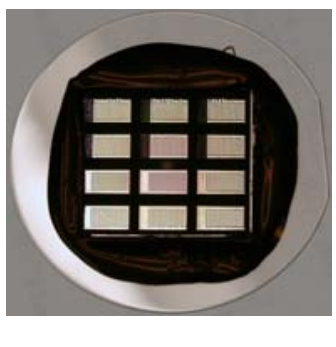

Fig. 4. The tubes are embedded in the wax (black) after the backing $\mathrm{Si}$ is etched.

a number of sources, and therefore, our work to date has focused on step three; a three dimensional micromachining process compatible with prefabricated CMOS circuits and pick and place components. In developing this process, we have chosen an approach based on stress driven selfassembly of three dimensional structures [14], [15].

The fabrication process used for the tubes is illustrated schematically in Fig. 3. It begins (1) by thermally growing a $2 \mu m$ thick layer of $\mathrm{SiO}_{2}$ on a silicon wafer. (2) $\mathrm{The}_{\mathrm{SiO}}$ is then patterned using buffered oxide etch (BOE) to form rectangular islands that will later become the tube microrobots. (3) Aluminum $\left(\mathrm{Al}_{0.99} \mathrm{CuSi}\right)$ of various thicknesses $(0.1 \mu \mathrm{m}$ to $0.5 \mu \mathrm{m}$ ) is sputtered onto the $\mathrm{SiO}_{2}$ and patterned using a lift off process. At this point, the materials required to form the tube are all in place and the next step is to free them from the original silicon wafer. In order to do this, it is necessary to protect the aluminum, any CMOS circuits, or mounted die from the silicon etch process. (4) Therefore, the wafer is mounted, device side down, onto a sapphire handle wafer using Apiezon Wax W [2]. This wax is very stable and is not significantly attacked during the remaining process steps. Once the devices are mounted on the handle wafer, (5) the back side of the silicon device wafer is etched. Since it is necessary to etch over $0.5 \mathrm{~mm}$ of silicon, an initial etch is done using HNA (HF-Nitric-Acetic Acid). When the device wafer thickness is below $100 \mu \mathrm{m}$, the rest of the etch is done using $\mathrm{XeF}_{2}$, an isotropic gas phase silicon etchant that does not significantly etch most other CMOS circuit materials including $\mathrm{SiO}_{2}, \mathrm{Al}$, or photoresist. The final $\mathrm{XeF}_{2}$ etch stops cleanly on the $\mathrm{SiO}_{2}$ and wax surfaces as shown in Fig. 4. (6) The devices are then released from the wax by immersion in trichloroethylene (TCE).

Upon release of the tubes from the wax (7), the rectangular shaped tubes bend into cylinders due to residual stresses in the $\mathrm{SiO}_{2}$ and $\mathrm{Al}$. During the $\mathrm{SiO}_{2}$ thermal growth process, oxygen atoms are introduced into a pre-defined silicon lattice $\mathrm{SiO}_{2}$. Not surprisingly, the resulting oxide layer is highly stressed ( $\approx 350 \mathrm{MPa}$, compressive). In contrast, the sputtered Al films can take on a wide range of stress values depending upon the deposition parameters. Under most conditions, these films will have tensile stress with a lower magnitude (typically $<60 \mathrm{MPa}$, tensile).

Upon release, the $\mathrm{SiO}_{2}$ expands more than the $\mathrm{Al}$, causing the rectangular structure to bend into a cylinder, with the Al on the inside. The physical shape of the oxide patch and the 


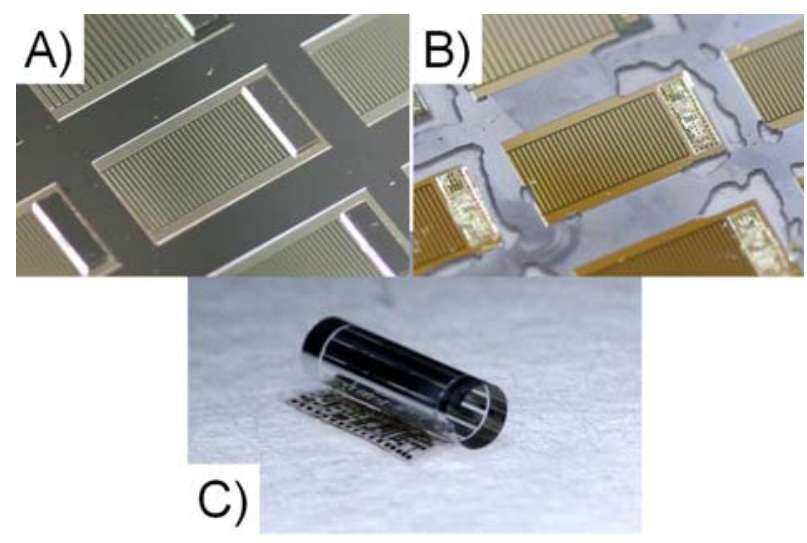

Fig. 5. A) After bonding the SOI CMOS chip to the tubes. B) After back-etching the chip. $C)$ A released tube which requires re-rolling.

pattern of the $\mathrm{Al}$ electrodes are used to control the direction of bending. In general, a rectangular oxide patch will bend so that the long direction rolls up leaving the shorter axis as the length of the tube.

As discussed in Section II-B, the radius of the tube depends heavily on the thickness of the layers. In general it is possible to have different thicknesses or patterns of aluminum on the same tube, to create parts with different radii. For example, in Fig. 4, the bottom six tubes have a solid aluminum patch on the left side, which, when released, bends with a smaller radius of curvature. By controlling the bending process in this way, it will be possible to mount a CMOS die on one end of the pre-released structure. During folding the region the die is bonded to will stay flat, then a second region starting at the edge of the chip will have a smaller radius of curvature, followed by an outer region with a larger radius of curvature (See Fig. 3 (8)). Properly controlling the radius of curvature in these two sections will enable us to keep the CMOS die close to the center of the tube. This is crucial since as discussed in Section III-B, a non-centered chip would make actuation more difficult.

To further improve actuation performance and reduce manufacturing difficulty we etch the back side silicon of the Silicon-on-insulator (SOI) CMOS die down to the $\mathrm{SiO}_{2}$ insulator layer after it is bonded to the tube (See Fig. 5). This substantially reduces the weight of the die and makes the entire structure smoother, enhancing the release process.

\section{B. Analysis of the Curvature}

The radius of curvature for the released tubes can be predicted using the methods described in [12] and [14]. In our case, we have a two layer structure composed of $\mathrm{SiO}_{2}$ and Al. Each layer has a thickness $t_{i}$, Young's modulus of $E_{i}$, a residual stress of $\sigma_{i}$. Fig. 6 shows calculated diameters for a range of layer thicknesses assuming $E_{A l}=70 \mathrm{GPa}$, $E_{\mathrm{SiO}_{2}}=60 \mathrm{GPa}, \sigma_{a l}=60 \mathrm{MPa}, \sigma_{\mathrm{SiO}_{2}}=-550 \mathrm{MPa}$.

We measured thicknesses of tubes with both solid (Fig. 6, circles) and patterned Aluminum (Fig. 6, crosses). The patterned tubes have $100 \mu \mathrm{m}$ wide stripes of Aluminum electrodes with a pitch of $100 \mu \mathrm{m}$, so the total aluminum coverage of the tube surface is $50 \%$.

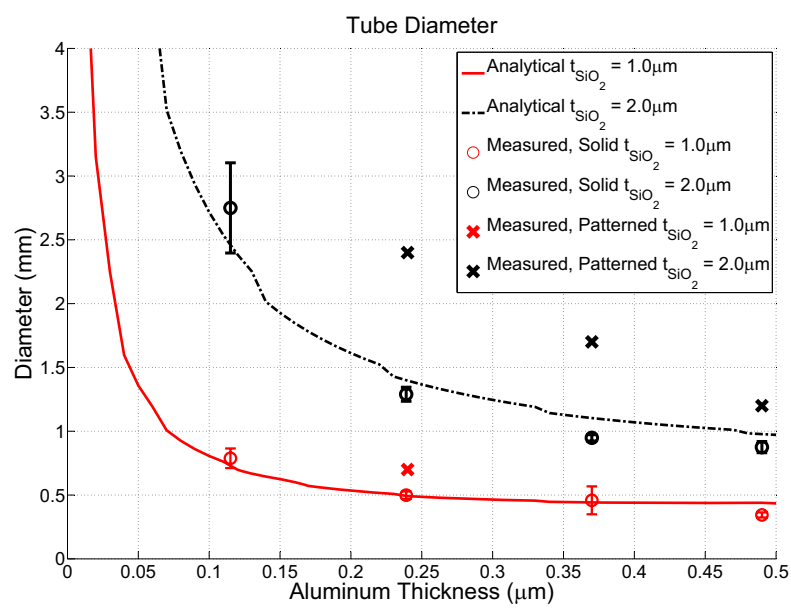

Fig. 6. Tube diameter for different thicknesses of $\mathrm{SiO}_{2}$ and Al.

We see that for $t_{\mathrm{SiO}_{2}}=2 \mu \mathrm{m}$ and $t_{\mathrm{Al}}=0.5 \mu \mathrm{m}$ the expected diameter of a solid $\mathrm{Al}$ tube is $0.97 \mathrm{~mm}$. We choose to use $t_{A l}=0.49 \mu \mathrm{m}$ and were able to achieve a diameter of $0.87 \mathrm{~mm}$. The same thicknesses resulted in a $1.2 \mathrm{~mm}$ tube when the aluminum is patterned. This increase in tube diameter is expected, as with less aluminum coverage, there is less stress and thus less bending, resulting in a larger diameter.

The fitted stress of $-550 M P a$ for the $\mathrm{SiO}_{2}$ is higher than the values typically reported for thermally grown $\mathrm{SiO}_{2}$ $(\approx-250 M P a$ to $-350 M P a)$. This is likely due to a combination of systematic measurement error and a stress gradient in the material.

As shown in Fig. 6, the diameters of the tubes we manufactured are in agreement with our analysis. Although thinner $\mathrm{SiO}_{2}$ gives a broader range of realizable diameters, the resulting tubes are too fragile. Using a thickness of $2 \mu \mathrm{m}$ yields robust tubes with useful diameters.

\section{ACTUATION}

An important design goal in realizing claytronics is to use a single mechanism to perform as many of the required functions as possible. The actuation mechanism for catoms is the most challenging of the functions; it is essential that catoms are able to move and reconfigure in an ensemble. In this section, we describe an actuation mechanism for catoms based on electrostatics. In Section IV, we describe how the same mechanism may be used to implement other functions.

Catoms move by rolling along the surface of neighboring catoms. They use electrostatic forces to do this. Each catom has conducting plates ("electrodes") covering its outer shell; they run along the length of the tube, e.g., in and out of the page in Fig. 7. The electrodes of neighboring catoms align and form capacitors (see Fig. 7). When a voltage is applied between the plates of a capacitor, attractive electrostatic forces are created due to the accumulated charge on the electrodes. These forces then cause the catom to move.

Since catoms are not electrically connected, they can not share a common ground. In order to generate a voltage across the electrodes, adjacent catoms are coupled using at least 


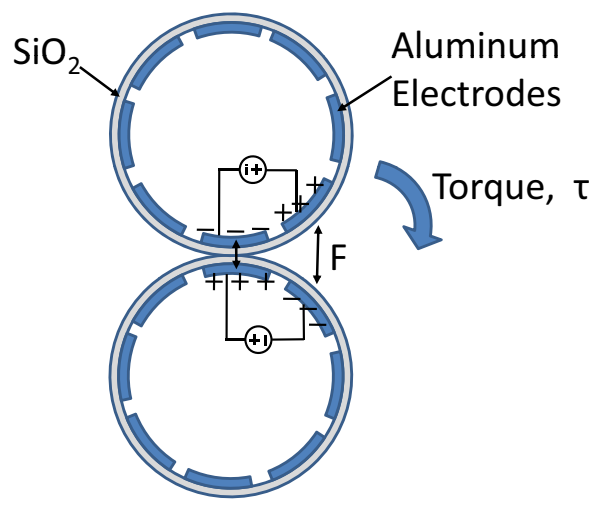

Fig. 7. The force created between the electrodes of adjacent catoms moves the catom.

two pairs of electrodes. This way, a series circuit loop is formed. The total electrostatic force created between the electrodes causes the catoms to roll around each other until an equilibrium is reached. By switching the voltage on the electrodes, and successively charging/discharging the next pair, a continuous rolling motion is possible.

The analysis in Section III-A estimates the forces created between catoms. In Section III-B we show that we can meet the challenge of actuating tubes even when the tubes are not perfect cylinders due to non-uniformity introduced by the manufacturing process.

\section{A. Force Created Based On Voltage}

Before we analyze how much force can be created, it is important to understand how much force is needed to actuate a catom.

For a catom to roll around a fixed catom, in the worst case, the torque created by the electrostatic forces must be enough to overcome the moment created by the weight of the catom, with respect to the point of contact. The moment created by the weight of a catom is:

$$
\tau_{\text {required }}=M g R=6.8 \times 10^{-10} \mathrm{Nm}
$$

where $R$ is the radius of the catom, $M$ is the total mass of the catom, including the chip. The tube dimensions are, diameter $2 \times R=1.2 \mathrm{~mm}$, length $L_{t u b e}=4 \mathrm{~mm}$ and thickness $t_{\mathrm{SiO}_{2}}=2 \mu \mathrm{m}$ for the $\mathrm{SiO}_{2}$ and $t_{A l}=0.5 \mu \mathrm{m}$ for the $\mathrm{Al}$. Using the densities of materials $\rho_{\mathrm{SiO}_{2}}=2330 \mathrm{~kg} / \mathrm{m}^{3}$ and $\rho_{A l}=2700 \mathrm{~kg} / \mathrm{m}^{3}$, the tube mass is $M_{\text {tube }}=8 \times 10^{-8} \mathrm{~kg}$. The dimensions of the chip are $H=15 \mu \mathrm{m}, L=900 \mu \mathrm{m}$, $w=1200 \mu \mathrm{m}$. The chip is a Silicon-on-insulator (SOI) CMOS with the backing silicon removed, and is assumed to have the density of Aluminum. Then, $M_{\text {chip }}=3.7 \times 10^{-8} \mathrm{~kg}$, and the total mass is $M=1.17 \times 10^{-7} \mathrm{~kg}$, resulting in the moment given in equation 1 .

As shown in Fig. 8, the electrodes are placed radially on a catom, each spanning an angle of $\theta_{w}$. Adjacent electrodes on the same catom are separated by a gap, so that they do not experience dielectric breakdown. In Fig. 8, when the catom on top is to roll clockwise around the bottom catom, a voltage difference is applied between all the electrodes that

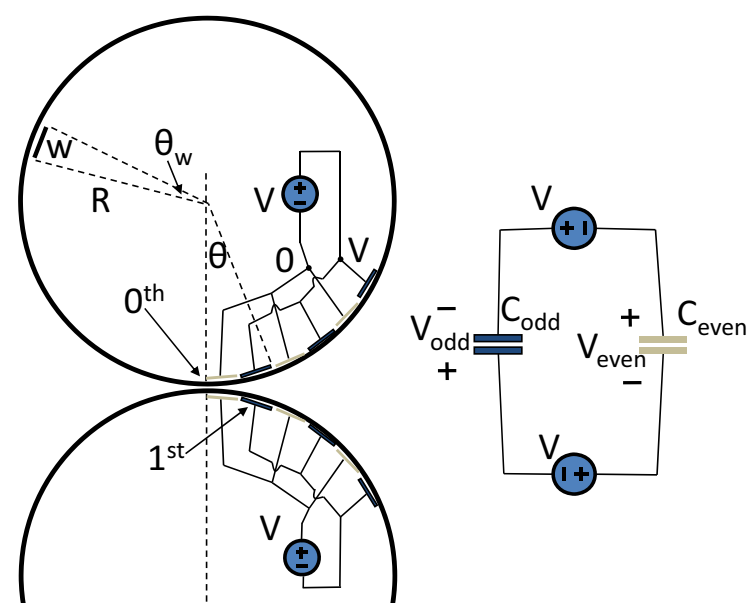

Fig. 8. The electrodes on neighboring catoms are paired to form capacitors.

are located in the bottom right quadrant of the top catom and the electrodes that are located at the upper right quadrant of the lower catom.

In this analysis, $n$ is the index of the electrode pair listed counter clockwise on the top catom, and clockwise on the bottom catom. The $n=0$ electrode pair is the one that is at the point of contact. The odd numbered pairs are connected in parallel to form $C_{o d d}=\sum_{i=o d d} C_{i}$ and the even numbered electrodes of the catoms are connected in parallel to form $C_{\text {even }}=\sum_{i=\text { even }} C_{i}$. The electrodes are connected in this alternating fashion to ensure that voltages across $C_{\text {odd }}$ and $C_{\text {even }}$ have nearly equal values. Since electrostatic force is proportional to the square of the voltage, the maximum force is created when the voltages on the two capacitors are equal.

The voltage drops across $C_{\text {odd }}$ and $C_{\text {even }}$ are, then:

$$
V_{\text {odd }}=\frac{2 V C_{\text {even }}}{C_{\text {odd }}+C_{\text {even }}}, \quad V_{\text {even }}=\frac{2 V C_{\text {odd }}}{C_{\text {odd }}+C_{\text {even }}}
$$

Our analysis is based on a parallel plate capacitor model for calculating the force and torque. This assumption is reasonable considering the fact that the major contribution to the force comes from the electrodes near the contact point, which are almost parallel. We calculate the voltage and force between the electrodes, and then find the torque with respect to the contact point.

For an infinitesimal electrode of length $d \theta R$ on the circumference of the tube, the electrostatic force is:

$$
d F=\frac{\epsilon_{0} L V_{n}^{2} \cos (\theta) R d \theta}{2\left(d_{0} / \cos (\theta)+2 R(1-\cos (\theta))\right)^{2}}
$$

where $V_{n}$ is the voltage across $n^{\text {th }}$ electrode pair, $R$ is the radius of the tube, and $L$ is the electrode length (along the length of the tube). The "dielectric" thickness of the $\mathrm{SiO}_{2}$ between the electrodes, $d_{0}$, is, $d_{0}=2 \times t_{S_{i 0_{2}}} / \epsilon_{r}$, twice the thickness of $\mathrm{SiO}_{2}$, for the two catoms, divided by the relative dielectric permittivity of $\mathrm{SiO}_{2}, \epsilon_{r} . \theta$ is the angle measured from the contact point. 


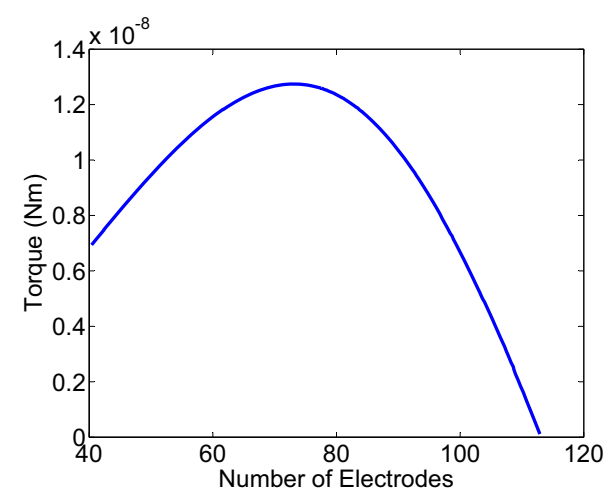

Fig. 9. Torque vs. Number of Actuation Electrodes.

The torque generated with respect to the point where the catoms come into contact is simply the force times the moment arm:

$$
d \tau=R \sin (\theta) d F
$$

It is possible to analytically calculate the total torque for given parameters. Fig. 9 shows the generated torque for a changing number of electrodes on the tube. This analysis was done for a catom of radius $R=0.6 \mathrm{~mm}$, electrode length $L=3.8 \mathrm{~mm}$, actuation voltage $V=100$ volts. $d_{0}=$ $2 \times t_{\mathrm{SiO}_{2}} / \epsilon_{r}=1.05 \mu \mathrm{m},\left(\epsilon_{r}=3.8\right)$. The maximum amount of torque is for 73 electrodes, and it is $\tau_{\text {total }}=1.28 \times 10^{-8} \mathrm{Nm}$. This analysis takes into account the fact that successive electrodes of the same catom can not be infinitely close to each other, otherwise they experience dielectric breakdown.

It is important to note that there is an optimal number of electrodes which maximizes the torque. Given a potential difference between successive electrodes, we obtain a minimum gap to avoid dielectric breakdown. When electrodes are smaller, more can be placed on the circumference, however, the ratio of electrode area to the catom surface is decreased due to the gap. This results in less capacitance and thus less overall force. When the electrodes are bigger, the difference between the capacitances of the odd and even numbered electrode pairs increases (see Fig. 8), resulting in different voltages across $C_{o d d}$ and $C_{\text {even }}$, and thus less force. For our calculation, we assumed that the gap is $100 \mathrm{~V} / E_{\text {air }}=$ $100 \mathrm{~V} / 3 \times 10^{6} \mathrm{~V} / \mathrm{m}=33 \mu \mathrm{m}$. $E_{\text {air }}$ is the dielectric strength of air.

The generated torque is greater than the worst case required torque required by a factor of 18 . Note that when the number of electrodes reaches 113 , the electrode area is reduced to zero.

\section{B. Required Voltage based on Eccentricity}

Since no fabrication technique is perfect, is it reasonable to expect the catom to have a nonuniform shape after the fabrication steps explained in Section II-A. In this section we investigate the effect of imperfect catom shape, and calculate the torque that is required in this case for a catom moving horizontally.

We model our catom as a tube with an elliptical cross section, with the major axis $a=1 \mathrm{~mm}$, and a variable

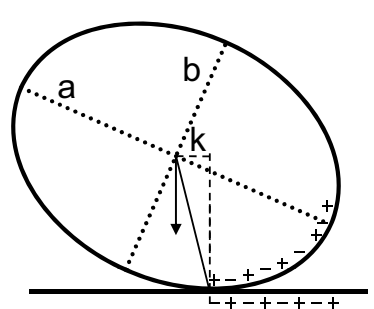

Fig. 10. Ellipse with the maximum moment arm. For $a: b=4: 3$, the corresponding eccentricity is 0.66 .

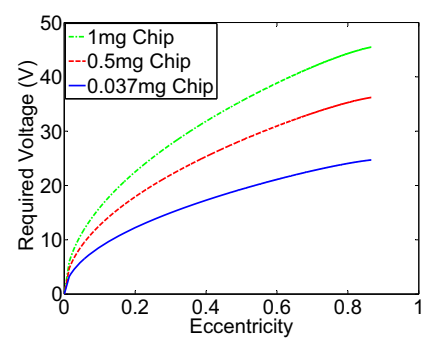

Fig. 11. The actuation voltage required to overcome the worst case moment due to eccentricity. minor axis, $b$. We define the eccentricity of the catom as: $e=\sqrt{1-b^{2} / a^{2}}$. Eccentricity is simply a measure of how distorted the catom is. With our current fabrication technique as described in Section II-A, we are able to fabricate tubes that have minor-to-major axis ratios ranging from 0.9 to 1 , corresponding to an eccentricity of $e=0.43$ to 0 .

Using an analysis similar to the one in Section III-A, a torque requirement is computed based on the eccentricity of catom. We find the biggest moment arm of the center of mass of the catom, with respect to the point of contact. Fig. 10 shows this moment arm, k, which can be calculated analytically as $k=\sqrt{(a-b) b a /(a+b)}$.

Given an eccentricity value, the minimum required torque for actuation is calculated, and compared to the torque generated as a function of voltage. Note that the electrostatic torque is also calculated by taking into account the deformed shape of the catom, which results in different capacitance distributions. Fig. 10 shows the actuation diagram in this case.

For the calculation, it is assumed that only the electrodes on a quadrant of the tube are energized. For different eccentricity values, we calculate the required voltage to overcome the requirement torque due to imperfect shape, as plotted in Fig. 11. The same graph is also plotted for different size chips, with the solid line being the voltage requirement for a chip with the mass $M_{\text {chip }}=3.7 \times 10^{-8} \mathrm{~kg}$, as calculated in Section III-A.

For the worst case measured eccentricity value of $e=$ 0.43 , the required voltage to overcome the torque due to the imperfect shape is $V=18$ volts. This is well below the operation voltage of the tube, $V=100 \mathrm{~V}$. Even if the chip size were to grow twenty fold, the required voltage would still be manageable.

We conclude that even in the worst case, it is possible to create a torque more than two times needed to actuate the tubes made using our fabrication technique which yields tubes with an eccentricity less than 0.43 .

\section{Electrostatic Simulations}

Using the commercial simulation package Comsol [4], we simulated the electrostatic force/torque. Fig. 12a shows the simulation of the case described in Section III-A, for $N=75$ electrodes and including a gap of $33 \mu \mathrm{m}$ between the electrodes. The tubes are aligned, and a potential difference 


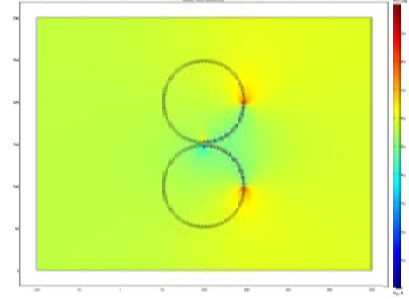

Fig. 12. Tubes actuated as described in Section III-A.

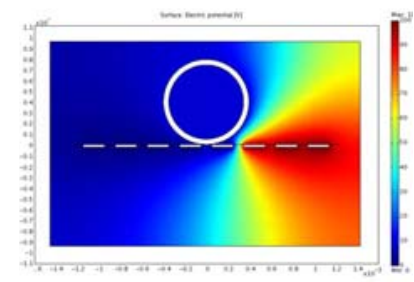

Fig. 13. Passive tube on electrode array. is created between the electrodes on the quadrants of the catoms.

The potential distribution and the surface charge density is calculated. The total torque on the top catom is calculated by integrating the electrostatic pressure over all surfaces of the tube:

$$
\int_{\text {tube }} d \tau=\int \frac{\sigma^{2}}{2 \epsilon_{0}}\left(\vec{r}-\overrightarrow{r_{0}}\right) \times d \vec{n}
$$

where $\sigma$ is the surface charge density, $d \vec{n}$ is the area vector normal to the surface, and $\overrightarrow{r_{0}}$ is the position of the contact point.

The total torque is $\tau_{\text {sim }}=3.68 \times 10^{-6} \mathrm{Nm} / \mathrm{m}$, directed into the plane. Note that the torque is per unit length of the tube, as the simulation is done on a cross section of the tube. For a tube that is $L=3.8 \mathrm{~mm}$, this corresponds to a torque of $\tau_{\text {sim }}=1.40 \times 10^{-8} \mathrm{Nm}$, compared to the theoretical value calculated as $1.28 \times 10^{-8} \mathrm{Nm}$.

We also simulated another case for actuation as shown in Fig. 13. In this case, the bottom catom is replaced by a flat plate with the electrodes, and the top catom is a conductive (equipotential) cylindrical surface. A potential difference is created between the electrodes next to the tube. We calculate the total torque in this case is $\tau=4.4 \times 10^{-5} \mathrm{Nm} / \mathrm{m}$. We also modified our analysis in Section III-A to calculate a theoretical value for this case, which is $\tau=3.1 \times 10^{-5} \mathrm{Nm} / \mathrm{m}$.

In both cases described in Fig. 12 and Fig. 13, the simulated torque is greater than the theoretical torque by factors of 1.09 and 1.41, respectively. This is mostly because the theoretical model is conservative, so it does not include effects like fringing fields. The simulation results are in agreement with our conclusion on the viability of actuation. Furthermore, in the real case, we expect to have local deformations of the tubes near the contact point due to the forces created. These deformations will increase the coupling between the tubes and thus will create more torque.

\section{Experiments}

In this section, we demonstrate an experimental setup where a cylindrical aluminum tube is actuated with the use of external electrostatic fields.

In this setup, aluminum electrodes are placed on a flat substrate, called the "stator". The electrodes are covered with a dielectric material (SU-8 2000, [13]). A single piece of aluminum tube with a diameter $D=1 \mathrm{~mm}$, made out of thin aluminum foil, is placed on top of the stator. The length of the tube runs along the electrodes underneath.
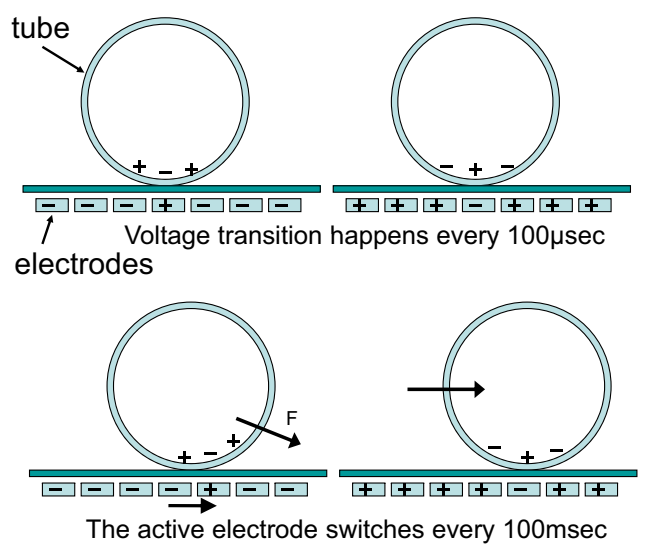

Fig. 14. The experimental setup of actuation.

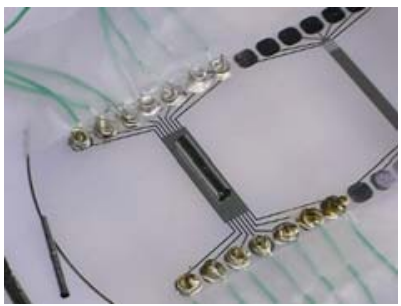

(a)

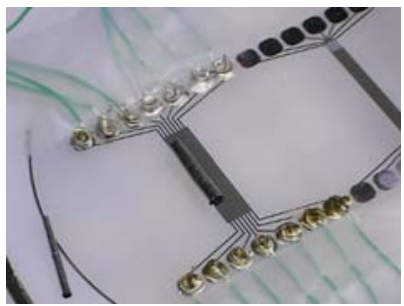

(b)
Fig. 15. The tube in motion. a) tube moving forward, b) tube moving backward. The video can be found at Http://www.cs.cmu.edu/ claytronics/movies/1x_tube_back-and-forth.mp4.

Fig. 14 shows the configuration of the experimental setup. The electrodes are placed on a fixed substrate. There are no patterned electrodes on the tube. The operation of this setup is similar to the linear electrostatic motor described in [8]. When a voltage difference is created between the electrodes of the stator, the electric fields create mirror charges on the tube. This charge distribution creates a net force toward an equilibrium point. As the tube starts to roll in the presence of this force, the voltage is switched to the next electrode, creating a continuous rolling motion.

This experimental configuration differs from the mechanism explained in Section III, by the fact that the electrodes are placed on an external substrate, the stator. However, the stator can be thought of as the neighboring catom. Therefore, this configuration is analogous to the one described, except for the fact that the top catom (in this case, the passive tube) does not have its own voltage source.

Fig. 15 shows still images from a video of the tube in motion [3]. An external microcontroller circuit creates an AC square-wave voltage as high as $200 \mathrm{~V}$ on successive electrodes. This voltage is significantly higher than the voltage discussed in Section III. However, the effective potential difference across the capacitors is half of the source voltage, since the tube is passive (has no voltage source). The polarity of the voltage is switched every $100 \mu \mathrm{sec}$ (see Fig. 14). At a given time, one electrode has a positive high voltage, and all others are grounded. At this moment, the static equilibrium point for the tube is exactly above this electrode. After $100 \mu \mathrm{sec}$, this electrode switches to ground, and all the other electrodes are switched to $200 \mathrm{~V}$. The active electrode is 
switched every $100 \mathrm{msec}$, setting a new equilibrium point for the tube, and causing it to roll.

This experimental setup demonstrates the viability of electrostatic forces as an actuation mechanism for catoms.

\section{Realizing The Ensemble Principle}

In the previous section we described the structures and methods for actuating catoms with electrostatic forces. Here we briefly describe how these same structures can be used for adhesion, communication, and power.

The capacitively coupled electrodes of adjacent catoms create electrostatic forces which are used for actuation and can clearly be used for adhesion, that is, when the catoms come to equilibrium, the capacitively coupled electrodes hold them in place. The use of capacitive coupling for inter-module latching was investigated in [11]. Our analysis showed that the bulk yield strength of an ensemble of catoms can be as high as that of plastics.

Power transfer is also possible using capacitively coupled electrodes. When one catom puts an AC signal on its half of the plates of the capacitors, it generates an AC signal on the adjacent plates of the neighboring catom. This signal can be rectified and converted to DC to power the neighboring catom. In the worse case we obtain power efficiencies of $50 \%$. In the best case, when the catoms are trickle charging each other we can achieve efficiencies of nearly $80 \%$.

Finally, communication using the same electrode pairs is even simpler. Data modulated with an AC signal can be easily transferred across the capacitors, and demodulated at the target.

\section{Realizing the CATOM}

There are many steps involved in realizing a working catom. To this point we have made progress in both manufacturing and actuation. The CMOS compatible fabrication process for forming three dimensional tubes from a two dimensional process has been developed to the point where tubes with two (or more) radii can consistently be fabricated at the same time, and even on the same die. The actuation process has been modeled and simulated allowing the actuator design to be optimized. Further, the simulations give us good confidence that the catoms can provide sufficient force to operate once they are implemented, even accounting for non-uniformities such as eccentricity. Proof of principle experiments have been done to demonstrate rolling tubes using external actuation. Therefore, the next step is to integrate active control circuits that collect and store energy and then use this energy to drive the catom.

Implementing the chip begins by selecting the appropriate CMOS foundry process. From the actuation design work, it is clear that standard CMOS will not work as we may need voltages of up to $100 \mathrm{~V}$ to drive the electrodes. Further, we would like a process that uses Silicon-on-insulator so that it can later be used as part of the shell forming process. Based on these constraints, we selected XFAB's XI10 High Voltage BCDMOS process [1] since it can support voltages in excess of $100 \mathrm{~V}$.

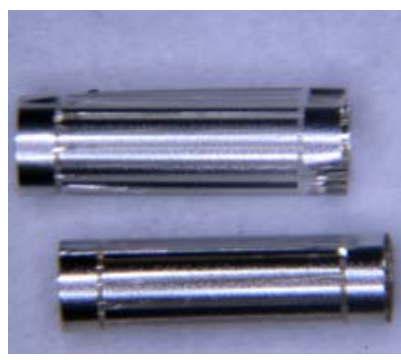

Fig. 16. Different radius tubes.

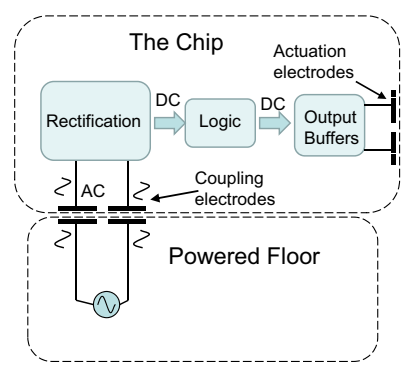

Fig. 17. The chip.
Fig. 17 shows the schematic diagram of the circuits required to implement the first catom. These circuits have all been to fit on a single die. The power required to operate the chip is supplied through capacitive coupling [11]. The tubes have electrodes running along the circumference on both ends, called the "coupling electrodes" (See Fig. 16). An AC signal generated at the source, i.e., a powered floor of another catom, is transfered to the chip through the coupling electrodes. These AC signals are rectified to generate a DC output. The DC power is then stored in a capacitor and used to power the integrated circuit and drive the electrodes at voltages of up to $100 \mathrm{~V}$.

When completed, this first prototype catom will demonstrate the viability of a mass-produced $\mathrm{mm}$-scale robot with internal control and the use of a single mechanism — external plates for capacitive coupling - which will be used for adhesion, actuation, and power transfer.

\section{REFERENCES}

[1] www.xfab.com/.

[2] Apiezon. Apiezon wax w, www.apiezon.com/pdfs/apwaxw.pdf.

[3] Claytronics at Carnegie Mellon University. www.cs.cmu.edu/_claytronics/movies/1x_tube_back-and-forth.mp4.

[4] Comsol. Comsol - multiphysics modeling, www.comsol.com/.

[5] B. Donald, C. Levey, et al. An untethered, electrostatic, globally controllable mems micro-robot. Microelectromechanical Systems, Journal of, 15(1):1-15, Feb. 2006.

[6] S. Floyd, C. Pawashe, et al. An untethered magnetically actuated micro-robot capable of motion on arbitrary surfaces. Robotics and Automation, 2008. ICRA 2008. IEEE International Conference on, pp. 419-424, May 2008.

[7] D. Frutiger, B. Kratochvil, et al. Magmites - wireless resonant magnetic microrobots. Robotics and Automation, 2008. ICRA 2008. IEEE International Conference on, pp. 1770-1771, May 2008.

[8] H. Fujita and A. Omodaka. The fabrication of an electrostatic linear actuator by silicon micromachining. Electron Devices, IEEE Transactions on, 35(6):731-734, Jun 1988.

[9] S. C. Goldstein and T. C. Mowry. Claytronics: A scalable basis for future robots. In RoboSphere 2004, November 2004.

[10] S. Hollar, A. Flynn, et al. Solar powered $10 \mathrm{mg}$ silicon robot. Micro Electro Mechanical Systems, 2003. MEMS-03 Kyoto. IEEE The Sixteenth Annual International Conference on, pp. 706-711, Jan. 2003.

[11] M. E. Karagozler, J. D. Campbell, et al. Electrostatic latching for inter-module adhesion, power transfer, and communication in modular robots. In Proceedings of the IEEE International Conference on Intelligent Robots and Systems (IROS '07), October 2007.

[12] C. Liu. In Foundations of MEMS, pp. 87-90, 2006.

[13] MicroChem. Microchem, www.microchem.com/products/pdf/su-8table-of-properties.pdf.

[14] J. Reid, V. Vasilyev, et al. Building micro-robots: A path to sub- $\mathrm{mm}^{3}$ autonomous systems. In Nanotech 2008, volume 3, 2008.

[15] V. Vasilyev, J. Reid, et al. Microfabrication of $\mathrm{si} / \mathrm{sio}_{2}$-spherical shells as a path to sub- $\mathrm{mm}^{3}$ autonomous robotic systems. In Proceedings of the Fall 2008 MRS Meeting, December 2008. 